\title{
EDITORIAL
}

\section{Anything you can do...}

Enzymes can serve as blueprints for artificial catalysts, the preparation of which may involve anything from biosynthesis of mutants to chemical synthesis of active site mimics.

The metabolic and growth rates of living organisms are sensitive to the regulation of enzyme expression, which is itself influenced by natural selection. Moreover, natural selection also shapes the enzymes themselves, the structures of which evolve to optimize functions that can be quantified in terms of kinetic and thermodynamic parameters. When encountering metalloenzymes for the first time, one is often led to believe that these biological catalysts have each evolved to a state of being 'perfect'. However, if selection pressure is weak - as is often the case for machinery involved in secondary metabolism - an enzyme may be far from 'optimal'. Although stronger selection pressure can afford faster enzymes, the potentially high activity of these enzymes in vivo may be difficult to recapitulate in another environment. Thus, when deploying an enzyme in a certain technology, it may be useful to specifically engineer or design one for this purpose.

When optimizing an enzyme's activity, a good starting point is to understand the interactions between the scaffold and any cofactors that it binds to. A Perspective by Anja Hemschemeier and Thomas Happe describes polypeptide-cofactor interactions in the case of redox metalloenzymes, the potentials of which can be sensitive to apparently minor structural changes such as solvation or hydrogen bonding of coordinated residues. If nature wants to tune these redox potentials even more dramatically, it can do so by choosing a different coordinating residue and/or metal ion. By analogy, in the lab it is often possible to perform site-directed mutagenesis and/or reconstitute the cofactor-free enzyme with an abiological metallocofactor. The latter approach affords spectacular results when applied to diiron hydrogenase, an enyzme that reversibly catalyses the interconversion $\mathrm{H}_{2} \rightleftharpoons 2 \mathrm{H}^{+}+2 \mathrm{e}^{-}-$a topical transformation in microorganisms and renewable energy technologies alike. It turns out that one can now reconstitute cofactor-free diiron hydrogenase with a variety of synthetic dinuclear iron complexes to give a family of semi-synthetic reconstituted enzymes, which one can study to systematically identify features that are important for catalysis.

Tweaking an existing enzyme in terms of amino acid sequence or cofactors may be considered a 'top-down' approach to enzyme engineering. Alternatively, one might start from scratch and attempt to mimic the function of a native polypeptide using a synthetic polypeptide scaffold. There is good reason to believe that such a de novo 'bottom-up' approach will afford active enzymes because the vast majority of sequence space is unexplored. For a given polypeptide length, the number of different amino acid sequences found in living systems is nowhere near the number of possible sequences. Adding synthetic amino acids and cofactors into the mix results in an even larger chemical space in which one might find a promising catalyst for biotic and, increasingly, abiotic reactions. Our ability to explore and exploit this space will depend on how well we can make use of empirical and computer-guided methods to identify useful and synthetically accessible sequences and cofactors.

The diiron hydrogenases are not the only enzymes that catalyse $\mathrm{H}_{2}$ production and oxidation. Indeed, despite being subject to different selection pressures, an older and phylogenetically unrelated class of enzymes also catalyses these reactions. We speak here of the nickel-iron hydrogenases, the active sites of which feature a different metal but operate according to the same mechanistic principles - each enzyme heterolyzes $\mathrm{H}_{2}$, making use of heteroatoms to relay protons and iron-sulfur clusters to relay electrons. Each active site is said to be poised in an 'entatic' state, a nowadays fashionable word used to describe an atomic arrangement that does not strongly bias one catalytic state over another. In this way, the respective activation energies are minimized - catalysis is a faster and less bumpy ride.

The most minimalistic 'bottom-up' approach to designing an artificial enzyme is to do away with most of the polypeptide and just use the active site cofactor or a close synthetic analogue. In many ways, this pursuit is to inorganic chemistry what natural product synthesis is to organic chemistry. However, chemists do not always choose this 'biomimetic' approach of copying an active site. Instead, using a bit of artistic license, they can opt for a 'bio-inspired' strategy, whereby they combine design features of enzymes with those of synthetic catalysts. The latter method has resulted in several success stories, notably the nickel phosphine-amine catalysts. These have diiron-hydrogenase-inspired amine proton relays proximal to a nickel centre ligated to phosphine donors - abiotic groups prominent in synthetic catalysts. One might thus consider these complexes to be synthetic mimics of both diiron and nickel-iron hydrogenases, and it is perhaps no surprise that nickel phosphine-amines can mediate, among other reactions, $\mathrm{H}_{2}$ oxidation and evolution. In their Perspective article, 
Arnab Dutta, Aaron Appel and Wendy Shaw pay particular attention to a privileged subset of these complexes that are electrochemically reversible. This is to say that these complexes, much like many hydrogenases, mediate $\mathrm{H}_{2}$ oxidation and evolution at the same potential and can thus enable substrates and products to equilibrate. This behaviour is rare for synthetic catalysts and is quite distinct from bidirectional irreversible catalysis, in which a catalyst can mediate forward and reverse reactions, but can only do so at different potentials because it requires an overpotential for at least one reaction. The logical conclusion is that the two catalytic reactions must proceed through distinct mechanisms - one cannot be the microscopic reverse of the other. Reversible and irreversible catalysts afford very different current responses, and the reversibility is only possible for nickel phosphineamines bearing an outer coordination sphere proton transfer chain (such as an amino acid-derived group) in addition to the amine proton relays situated in the second coordination sphere of the metal centre. Thus, irrespective of whether a design strategy involves mutating an enzyme from the top down or building one from the bottom up, it is often the case that we must have appropriate functionality in the first, second and even the outer coordination spheres.

Although we have so far concerned ourselves with proteic enzymes, we should also make mention of catalytic nucleic acids. The discovery of naturally occurring RNA catalysts (ribozymes) and DNA catalysts (DNAzymes) prompted many to subscribe to a 'RNA world hypothesis', which posits that RNA had not only a genetic role but also a catalytic one in the origins of life. The unique structural properties of nucleic acids are well known in the biomaterials community, and the propensity of certain sequences to form proteinlike cavities has attracted the attention of chemists and resulted in the design of artificial enzymes such as the Diels-Alder ribozyme. Even though the library of natural nucleic acid building blocks is smaller than that of amino acids, there are useful in vitro techniques to screen vast libraries of DNA and RNA molecules to assess the binding of these 'aptamers' to a given target molecule. When the target is a redox-active metal cofactor, one may thus hope to arrive at redox DNAzymes and ribozymes, which contrast with native nucleic acid catalysts because the latter typically only perform hydrolytic reactions. For example, one can now prepare RNA that stabilizes a photoexcited ruthenium complex and DNA to host to an active porphyrinatoiron oxidation catalyst. These systems represent encouraging evidence that the field of synthetic nucleic acid redox catalysis has arrived and is here to stay.

From directed evolution to laboratory synthesis, there are now many ways to construct and engineer artificial enzymes. Armed with mechanistic insights and design principles from both natural and synthetic systems, diverse scientific communities are participating together in a true confluence of research. It is exactly this contemporary and inclusive attitude that we hope to cultivate at Nature Reviews Chemistry. 